\title{
Diagnostic accuracy of diffusion-weighted imaging with conventional MR imaging for differentiating complex solid and cystic ovarian tumors at $1.5 \mathrm{~T}$
}

\author{
Ping Zhang ${ }^{1+}$, Yanfen $\mathrm{Cui}^{2 \dagger}$, Wenhua $\mathrm{Li}^{2^{*}}$, Gang Ren ${ }^{2}$, Caiting Chu ${ }^{2}$ and Xiangru $\mathrm{Wu}^{3}$
}

\begin{abstract}
Background: Preoperative characterization of complex solid and cystic adnexal masses is crucial for informing patients about possible surgical strategies. Our study aims to determine the usefulness of apparent diffusion coefficients (ADC) for characterizing complex solid and cystic adnexal masses.
\end{abstract}

Methods: One-hundred and 91 patients underwent diffusion-weighted (DW) magnetic resonance (MR) imaging of 202 ovarian masses. The mean ADC value of the solid components was measured and assessed for each ovarian mass. Differences in ADC between ovarian masses were tested using the Student's $t$-test. The receiver operating characteristic (ROC) was used to assess the ability of ADC to differentiate between benign and malignant complex adnexal masses.

Results: Eighty-five patients were premenopausal, and 106 were postmenopausal. Seventy-four of the 202 ovarian masses were benign and 128 were malignant. There was a significant difference between the mean ADC values of benign and malignant ovarian masses $(p<0.05)$. However, there were no significant differences in ADC values between fibrothecomas, Brenner tumors and malignant ovarian masses. The ROC analysis indicated that a cutoff ADC value of $1.20 \times 10^{-3} \mathrm{~mm}^{2} / \mathrm{s}$ may be the optimal one for differentiating between benign and malignant tumors.

Conclusions: A high signal intensity within the solid component on T2WI was less frequently in benign than in malignant adnexal masses. The combination of DW imaging with ADC value measurements and T2-weighted signal characteristics of solid components is useful for differentiating between benign and malignant ovarian masses.

Keywords: Ovary, Ovarian tumors, Diffusion-weighted imaging, Apparent diffusion coefficients

\section{Background}

Ovarian tumors are the leading indication for gynecologic surgery, and the preoperative characterization of complex solid and cystic adnexal masses is crucial for informing patients about possible surgical strategies. Recently, in order to improve quality of life, laparoscopic surgery has been increasingly used for the treatment of ovarian tumors that are believed to be benign. For malignant ovarian lesions, there is no evidence that laparoscopy for the

\footnotetext{
*Correspondence: wenhualimyj@sohu.com

${ }^{\dagger}$ Equal contributors

${ }^{2}$ Department of Radiology, Xinhua Hospital affiliated to Shanghai JiaoTong University School of Medicine, Shanghai 200092, China

Full list of author information is available at the end of the article
}

management of early stage ovarian tumors may improve the quality of life [1-3].

Ultrasonography (US) is the first-line imaging modality for adnexal lesions and is a particularly useful preoperative test for the characterization of noncomplex masses. Magnetic resonance imaging (MRI) may be of great help in identifying malignant lesions before surgery, particularly when US findings are suboptimal or indeterminate [4-10]. MRI can reveal morphologic characteristics such as papillary projections, nodularity, septa, solid portions and signal intensity on T1- and T2-weighted images, but none of these criteria reliably distinguish between benign and malignant tumors. The use of magnetic resonance (MR) diffusion-weighted imaging (DWI) may improve

\section{Biomed Central}

(c) 2012 Zhang et al.; licensee BioMed Central Ltd. This is an Open Access article distributed under the terms of the Creative Commons Attribution License (http://creativecommons.org/licenses/by/2.0), which permits unrestricted use, distribution, and reproduction in any medium, provided the original work is properly cited. 
MR characterization of ovarian lesions. Diffusionweighted imaging is sensitive to changes in the microdiffusion of water into both intracellular and extracellular spaces. Differences in the apparent diffusion coefficient (ADC) of benign and malignant complex adnexal masses have been reported [11-13]. Kayayama et al. [11] and Fujii et al. [14] assessed the feasibility of DWI for the differentiation of benign and malignant ovarian lesions. They concluded that the ADC values of cystic and solid components were not useful for differentiating between lesions. However, their data included endometrial cysts, mature cystic teratomas, and fibromas and fibrothecomas, in which hemorrhagic contents, sebaceous materials and fibrous tissue may cause an increase or a reduction in signal on DWI.

The purpose of this study was to clarify the relationship between the ADC values of the solid components of benign and malignant ovarian lesions, and to evaluate the supplementary use of ADC values of the solid components for differentiating between benign and malignant lesions.

\section{Methods}

Patients

Our institutional review board approved the study and waived the requirement to obtain written informed consent. A total of 601 female patients evaluated for ovarian tumors between January 2005 and December 2011 were enrolled. A retrospective review of MRI data was undertaken. The MRI studies met the following inclusion criteria for the study: (1) MRI was performed using a 1.5T magnet, and (2) both conventional MRI with DWI and dynamic contrasted-enhancement MRI were performed. In addition, the diagnosis was confirmed by surgery and pathological examination. Of the 601 patients, 383 were excluded due to the presence of endometriomas $(n=73)$, mature cystic or immature teratomas $(\mathrm{n}=39)$, and purely cystic ovarian lesions (including simple cysts and purely cystic adenomas) with thin and regular walls and/or septa $(n=232)$. A further 27 were excluded due to failed fat suppression or artifacts. The remaining 191 patients with 202 complex adnexal masses were included in the study.

\section{MR protocol}

All subjects underwent MRI with a $1.5 \mathrm{~T}$ MR unit (Twinspeed, GE Medical Systems, Milwaukee, WI, USA). The imaging protocol involved axial non-contrast T1-weighted (TR/TE, 400-600/10-14 ms) and axial T2-weighted (TR/TE, 4,000-6,000/100-120 ms) imaging performed with a chemical shift-selective fat saturation pulse using the following parameters: slice thickness, 5 $\mathrm{mm}$; gap, $1 \mathrm{~mm}$; field of view (FOV), 32 to $42 \mathrm{~cm}$; matrix, $256 \times 256$; and excitation, 2. Sagittal T1-weighted and T2-weighted (TR/TE, 3,000-6,000/100-110 ms) fast spin- echo imaging without chemical shift-selective fat saturation pulse was also performed, as well as post-contrast enhanced axial and sagittal T1-weighted imaging using parameters as described above, apart from a slice thickness of $5 \mathrm{~mm}$. Diffusion-weighted MRI was acquired in the axial plane prior to administration of contrast medium using a single-shot echo-planar imaging sequence (TR/TE effective range, 8,000-10,000/70-100; slice thickness/intersection gap, 5/1 mm; FOV, 32 to 42 $\mathrm{cm}$; matrix, $128 \times 128$; excitation, 2 . A b-value of 0 and of $1,000 \mathrm{~s} / \mathrm{mm}^{2}$ was also applied in three orthogonal (Z, Y, and $\mathrm{X})$ directions.

\section{MRI analysis}

Conventional MRI and DWI imaging data were analyzed on an Advantage Windows workstation 4.2 (GE Healthcare, Milwaukee, WI, USA) by two radiologists (with 15- and 10- year experience in pelvic MRI, respectively), in consensus, having carefully reviewed all images. The solid component, according to a previously established classification by Timmerman et al. [7] included thickened septa, vegetation (papillary projection) and solid portions that showed enhancement post-injection. The cystic component was defined as tissue with homogeneous long T1 and T2 characteristics or different signal intensities on T1- or T2-weighted MR images, and showed no enhancement post-injection. The signal intensity of solid components on T2-weighted MR images was defined as low or intermediate (equal to or greater) compared to that of the outer myometrium. The signal intensity of the cystic and solid components on DWI at b = $1,000 \mathrm{~s} / \mathrm{mm}^{2}$ was classified as intermediate or low compared to that of serous fluid (urine or cerebrospinal fluid).

\section{Data calculation and analysis}

The solid components of the lesions were identified on T2-weighted and post-contrast T1-weighted images, and were matched on ADC maps. The ADC values of the solid components of each tumor were measured on DW images by a radiologist using an Advantage Windows workstation 4.2 (GE Healthcare), using the manufacturer's software (FuncTool; GE, Medical Systems, Milwaukee, WI, USA). In order to minimize variability, the largest possible regions of interest (ROIs), which varied from 15 to $150 \mathrm{~mm}^{2}$, were manually placed in the solid parts of the tumor. When the lesion exhibited irregular or heterogeneous solid components, numerous vegetations or thickened irregular septa, between two and five ROIs were drawn within the targeted components and the mean ADC value was used in the analysis.

\section{Statistical analysis}

Surgical pathological findings served as the reference standard for assessment of ovarian tumors. All analyses 
were performed using SPSS version 13.0 for Windows (SPSS, Chicago, IL, USA). Differences in mean tumor $\mathrm{ADC}$ values between benign and malignant groups were evaluated using Student's $t$-test. A $P$-value $<0.05$ was considered statistically significant. Receiver operating characteristic (ROC) curve analysis was performed in order to assess the diagnostic performance of the mean $A D C$ values in terms of characterization of benign and malignant ovarian tumors.

\section{Results}

\section{Clinical demographics}

The surgical pathological types of 202 complex adnexal masses in 191 patients can be seen in Table 1. The mean patient age was $56.52 \pm 15.31$ (mean $\pm \mathrm{SD}$; range, 12 to $85)$ years. Eighty-five women $(44.5 \%)$ were premenopausal and 106 (55.5\%) were postmenopausal. Seventy-four (36.6\%) of the 202 ovarian masses were benign, and 128 (63.4\%) were malignant.

\section{MRI findings}

A high signal intensity within the solid component on T2-weighted MR images was observed less frequently in benign (39/74) than in malignant (108/128) adnexal masses $(P<0.01)$. The solid portion of 74 benign complex adnexal lesions contained homogeneous or heterogeneous low signal intensity on T2-weighted images in 100\% of cystadenofibromas (Figure 1), 83.3\% of fibrothecomas (Figure 2), 80.0\% of Brenner tumors, $23.5 \%$ of mucinous cystadenomas, and $21.4 \%$ of serous cystadenomas (Figure 3). Among 128 malignant complex adnexal masses, only 20 (15.6\%) displayed a low signal intensity of their solid components on T2-weighted images (Figure 4). On DWI, a high signal intensity at $\mathrm{b}=1,000 \mathrm{~s} / \mathrm{mm}^{2}$ within the solid component was observed less frequently in benign $(71.6 \%)$ than in malignant $(100 \%)$ adnexal masses $(P<0.05)$. Our results demonstrate that the following factors can be considered predictive of malignancy: the presence of a solid component with high or low signal intensity on T2-weighted images and high signal intensity on DWI with low ADC values (less than $1.20 \times 10^{-3} \mathrm{~mm}^{2} / \mathrm{s}$ ) at $\mathrm{b}=1,000 \mathrm{~s} / \mathrm{mm}^{2}$. On the other hand, the following factors can be considered predictive of a benign mass: the presence of a solid component with high or low signal intensity on T2-weighted images and high signal intensity on DW images with high $\mathrm{ADC}$ values (greater than $1.20 \times 10^{-3} \mathrm{~mm}^{2} / \mathrm{s}$ ), or low signal intensity on T2-weighted images and DW images with lower $A D C$ values at $b=1,000 \mathrm{~s} / \mathrm{mm}^{2}$.

\section{ADC analysis}

The mean ADC values of the tumor solid components were determined for each group. There was much overlap between the range of values observed for benign and for malignant tumors. However the mean ADC value for benign tumors was $1.22 \pm 0.46 \times 10^{-3} \mathrm{~mm}^{2} / \mathrm{s}$, and for malignant tumors $0.91 \pm 0.20 \times 10^{-3} \mathrm{~mm}^{2} / \mathrm{s}$, which was a significant difference $(P<0.05)$. This result suggests that an $\mathrm{ADC}$ value $\geq 1.20 \times 10^{-3} \mathrm{~mm}^{2} / \mathrm{s}$ may be the optimal cutoff for differentiating between benign and malignant tumors, with a sensitivity of $66.7 \%$, a specificity of $90.9 \%$, a positive predictive value (PPV) of $81.4 \%$, a negative predictive value (NPV) of $82.1 \%$, and an area under the curve (AUC) of 0.72. In our study, the mean ADC

Table 1 Histological types and apparent diffusion coefficient (ADC) values of 202 ovarian masses

\begin{tabular}{|c|c|c|c|c|c|}
\hline Type of ovarian mass & Lesions, number & High SI on T2-WI & High SI on DWI & Range of $A D C$ values & Mean ADC values \\
\hline Benign ovarian lesions & 74 & $39(52.7)$ & $53(71.6)$ & $0.12,1.81$ & $1.22 \pm 0.46$ \\
\hline Serous cystadenoma & 28 & $22(78.6)$ & $28(100)$ & $1.23,1.81$ & $1.52 \pm 0.19$ \\
\hline Mucinous cystadenoma & 17 & $13(76.5)$ & $17(100)$ & $1.12,1.79$ & $1.48 \pm 0.20$ \\
\hline Fibrothecoma & 18 & $3(16.7)$ & $3(16.7)$ & $0.40,1.58$ & $0.82 \pm 0.44$ \\
\hline Cystadenofibroma & 6 & $0(0)$ & $0(0)$ & $0.12,0.94$ & $0.52 \pm 0.26$ \\
\hline Brenner tumor & 5 & $1(20)$ & $5(100)$ & $0.88,1.44$ & $1.06 \pm 0.23$ \\
\hline Malignant ovarian lesions & 128 & $108(84.4)$ & $128(100)$ & $0.56,1.45$ & $0.91 \pm 0.20$ \\
\hline Serous cystadenocarcinoma & 35 & $26(74.3)$ & $35(100)$ & $0.66,1.35$ & $0.97 \pm 0.20$ \\
\hline Mucinous cystadenocarcinoma & 27 & $20(74.1)$ & $20(100)$ & $0.65,1.31$ & $0.89 \pm 0.19$ \\
\hline Serous borderline tumor & 12 & $10(83.3)$ & $10(100)$ & $0.78,1.45$ & $1.05 \pm 0.19$ \\
\hline Mucinous borderline tumor & 9 & $8(88.9)$ & $9(100)$ & $0.75,1.36$ & $0.99 \pm 0.17$ \\
\hline Clear cell adenocarcinoma & 8 & $8(100)$ & $8(100)$ & $0.69,1.01$ & $0.82 \pm 0.13$ \\
\hline Endometrioid adenocarconoma & 6 & $6(100)$ & $6(100)$ & $0.71,1.06$ & $0.93 \pm 0.11$ \\
\hline Granulosa cell tumor & 7 & $6(85.7)$ & $6(100)$ & $0.79,1.33$ & $0.98 \pm 0.18$ \\
\hline Undifferentiated adenocarcinoma & 5 & $5(100)$ & $5(100)$ & $0.64,1.19$ & $0.83 \pm 0.23$ \\
\hline Metastatic tumors & 19 & $19(100)$ & $19(100)$ & $0.56,1.09$ & $0.86 \pm 0.15$ \\
\hline
\end{tabular}

Numbers in parentheses are percentages; $\mathrm{ADC}$ values, (mean $\left.\pm \mathrm{SD} \times 10^{-3} \mathrm{~mm}^{2} / \mathrm{s}\right) ; \mathrm{T} 2-\mathrm{Wl}$, T2-weighted image; Sl, signal intensity; DWI, diffusion-weighted imaging. 

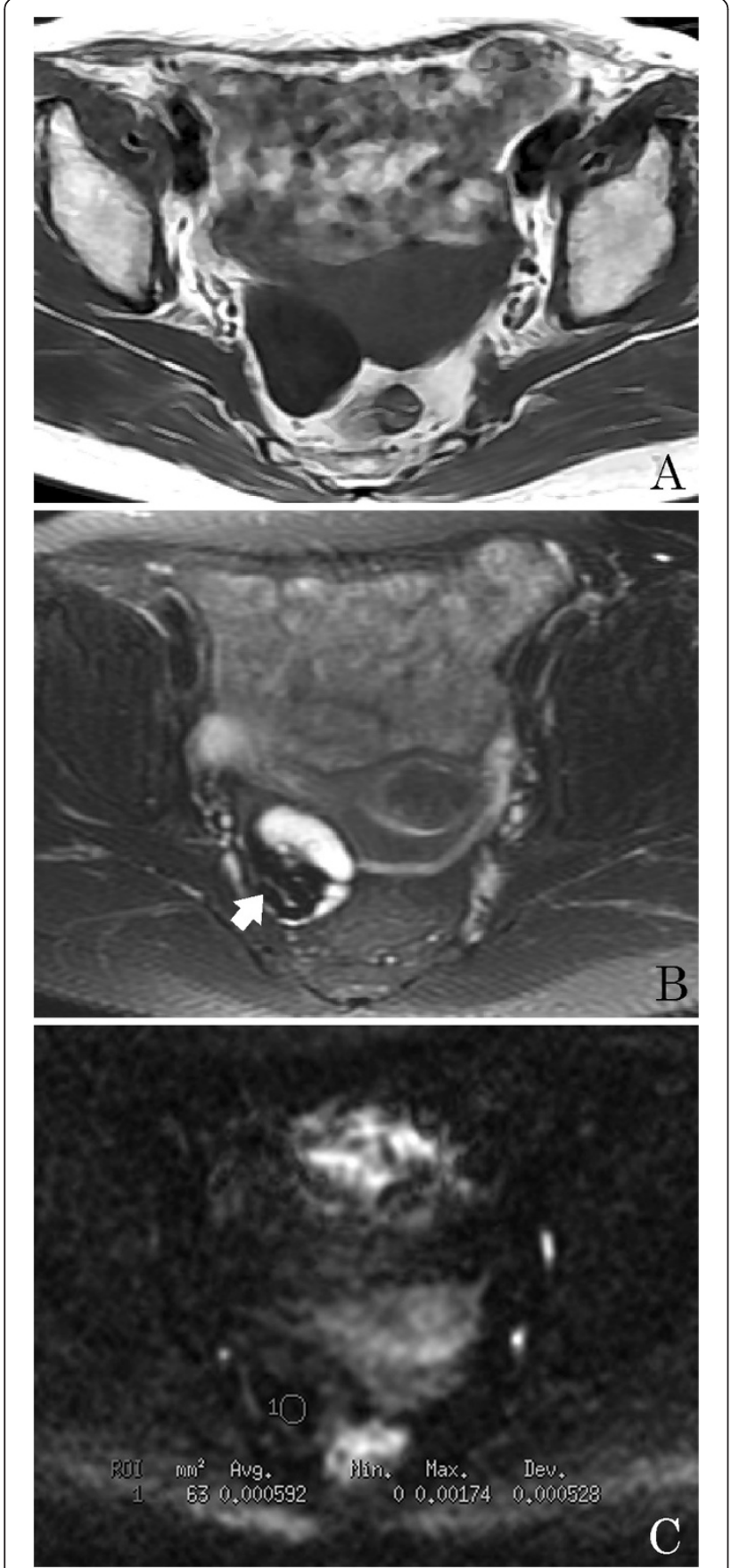

Figure 1 A 67-year-old woman with an ovarian cystadenofibroma. (A) Axial T1-weighted image shows a right ovarian mass with a hypointensity. (B) Axial T2-weighted image reveals that the solid part of the mass is hypointense (arrow) and the cystic component is hyperintense. (C) Axial diffusion-weighted imaging (DWI) reveals that the solid part of the mass has a very low signal intensity (circle 1, apparent diffusion coefficient $\left.(A D C)=0.592 \times 10^{-3} \mathrm{~mm}^{2} / \mathrm{s}\right)$. values were much lower for cystadenofibromas, fibrothecomas and Brenner tumors. There was no statistically significant difference between values for fibrothecomas, Brenner tumors and malignant tumors $(P>0.05)$, but there was a significant difference between values observed for cystadenofibromas and for malignant tumors $(P=0.002)$. When cystadenofibromas, fibrothecomas and Brenner tumors were excluded from the analysis, the sensitivity, specificity, PPV, NPV, and AUC for the optimal cutoff ADC value of $1.20 \times 10^{-3} \mathrm{~mm}^{2} / \mathrm{s}$ for differentiating between benign and malignant tumors, were $97.7 \%, 90.1 \%, 86.6 \%, 99.1 \%$, and 0.96 , respectively (Figure 5).

\section{Discussion}

Our findings demonstrate that the presence of high signal intensity in solid components of ovarian lesions on DW and T2-weighted imaging combined with low ADC values can be used to distinguish malignant from benign ovarian lesions. The results also suggest a potential role for DW imaging with quantitative analysis of ADC values in improving the diagnostic performance of ovarian MRI and yielding functional measures of the tumor microenvironment.

In the present study, $\mathrm{ADC}$ values are largely proportional to the ratio of extracellular and intracellular components, cell density, intracellular organelles, matrix fibers, and soluble macromolecules. Although some overlap in ADC values was observed between the benign and malignant groups, the mean ADC value of the 128 malignant ovarian tumors was significantly lower than that of the 74 benign ovarian tumors $(P<0.05)$. Our results suggest that an ADC value $\geq 1.20 \times 10^{-3} \mathrm{~mm}^{2} / \mathrm{s}$ may be the optimal cutoff for differentiating between benign and malignant tumors. Furthermore, a sensitivity of $66.7 \%$, a specificity of $90.9 \%$, a PPV $81.4 \%$, an NPV of $82.1 \%$, and an AUC of 0.72 , was observed with this ADC cutoff value. This result is consistent with previous reports $[10,12,15,16]$. In a study by Fujii et al. [14], the authors evaluated the contribution of DWI in combination with quantitative $\mathrm{ADC}$ analysis to the characterization of 123 ovarian lesions, which included 42 malignant and 81 benign lesions (including 7 fibromas, 18 mature cystic teratomas and 24 endometriomas). The results suggest that DW imaging of ovarian lesions and ADC values of the solid component are not useful for differentiating between benign and malignant ovarian lesions. This apparent discrepancy is probably due to the pathologic architectures of benign tumors. In our series, $83.3 \%$ (15/18) of fibrothecomas, $100 \%(6 / 6)$ of cystadenofibromas and $80 \%(4 / 5)$ of Brenner tumors demonstrated low signal intensity in the solid components on DWI and low ADC values, due to the presence of abundant collagen-producing 


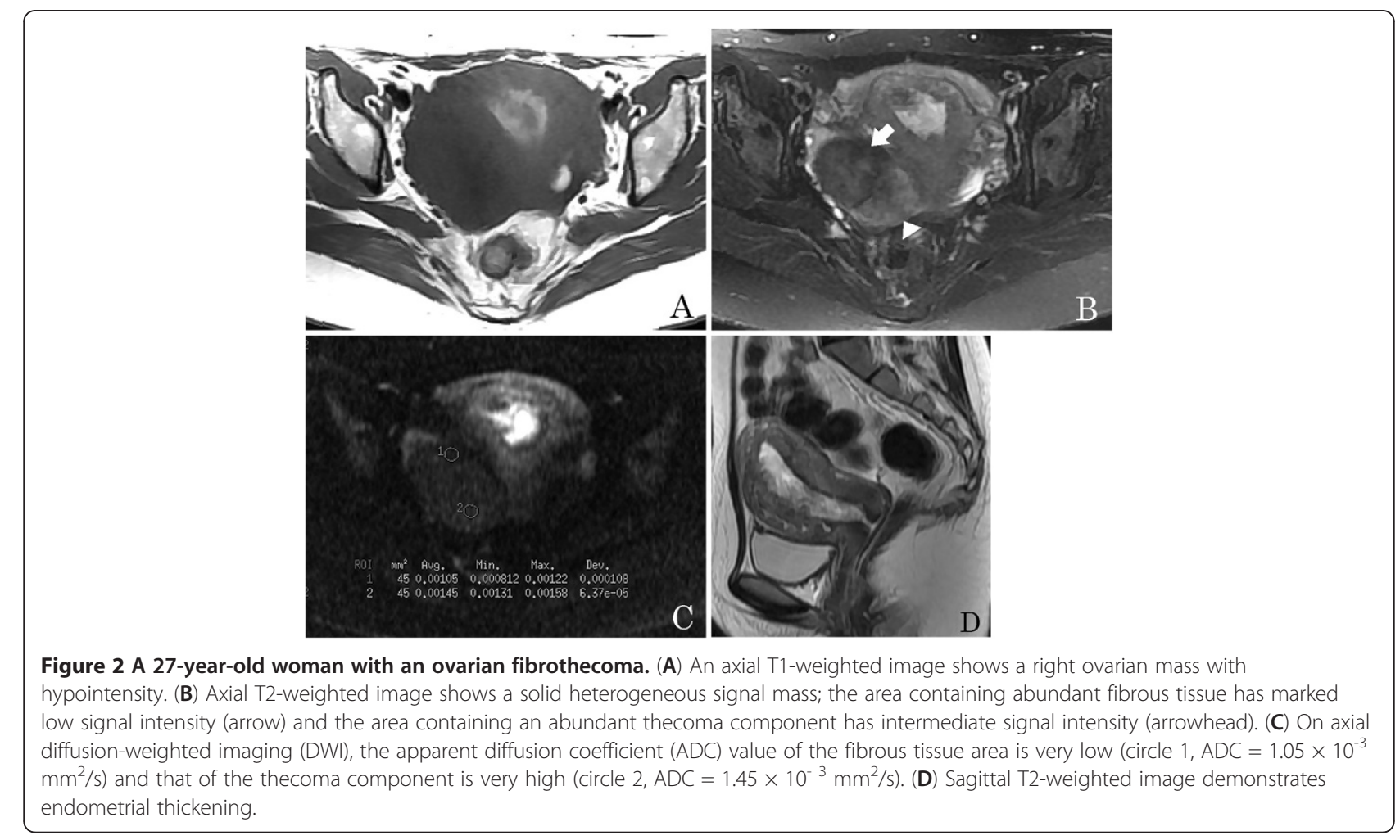

fibroblastic cells and a dense network of collagen fibers within the extracellular matrix, which can restrict the Brownian motion of water molecules. Previous studies have also reported that endometriomas and mature teratomas exhibit low ADC values [11]. This could explain the observed absence of a difference between the mean ADC values of benign and malignant ovarian lesions in the study by Fujii et al. [14].

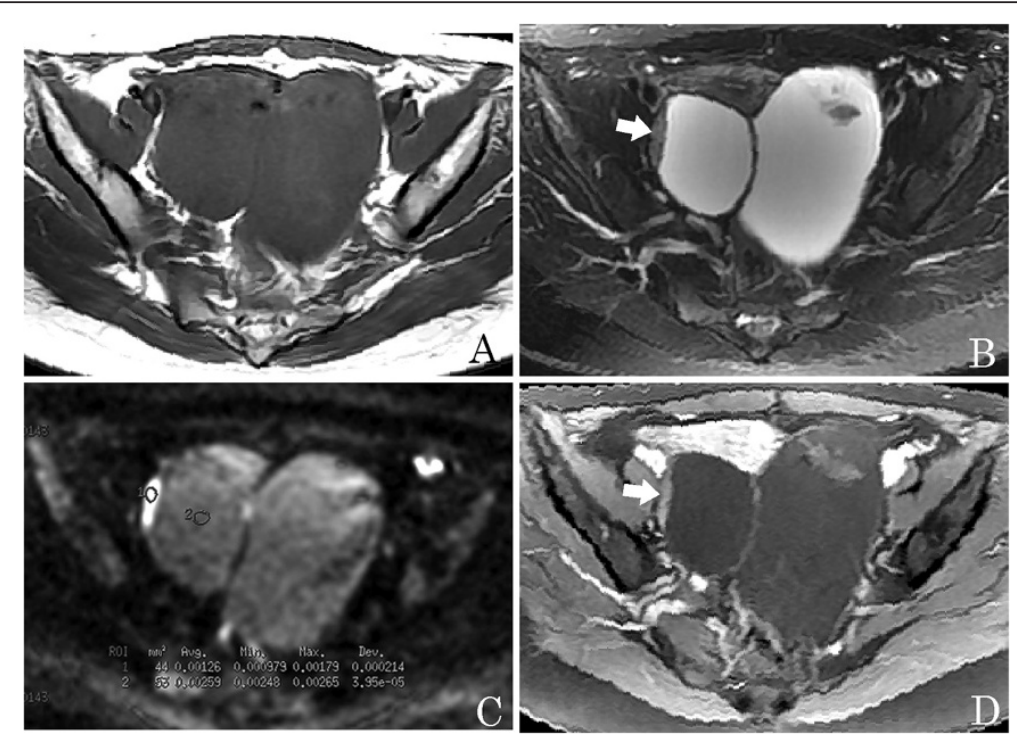

Figure 3 A 28-year-young woman with bilateral benign serous ovarian cystadenomas. (A) An axial T1-weighted image shows a pelvic tumor with hypointensity. (B) Axial T2-weighted image shows a complex adnexal tumor with solid and cystic components, and solid components as vegetations with intermediate signal intensity (arrow). (C) Axial diffusion-weighted imaging (DWI) obtained at $\mathrm{b}=1,000 \mathrm{~s} / \mathrm{mm}^{2}$ shows the solid component as hyperintense with a high apparent diffusion coefficient (ADC) value (circle $1, A D C=1.26 \times 10^{-3} \mathrm{~mm}^{2} / \mathrm{s}$ ), and the cystic component with an even higher value (circle 2, ADC $=2.59 \times 10^{-3} \mathrm{~mm}^{2} / \mathrm{s}$ ). (D) Axial contrast-enhanced T1-weighted image demonstrates slight enhancement of the solid component (arrow). 

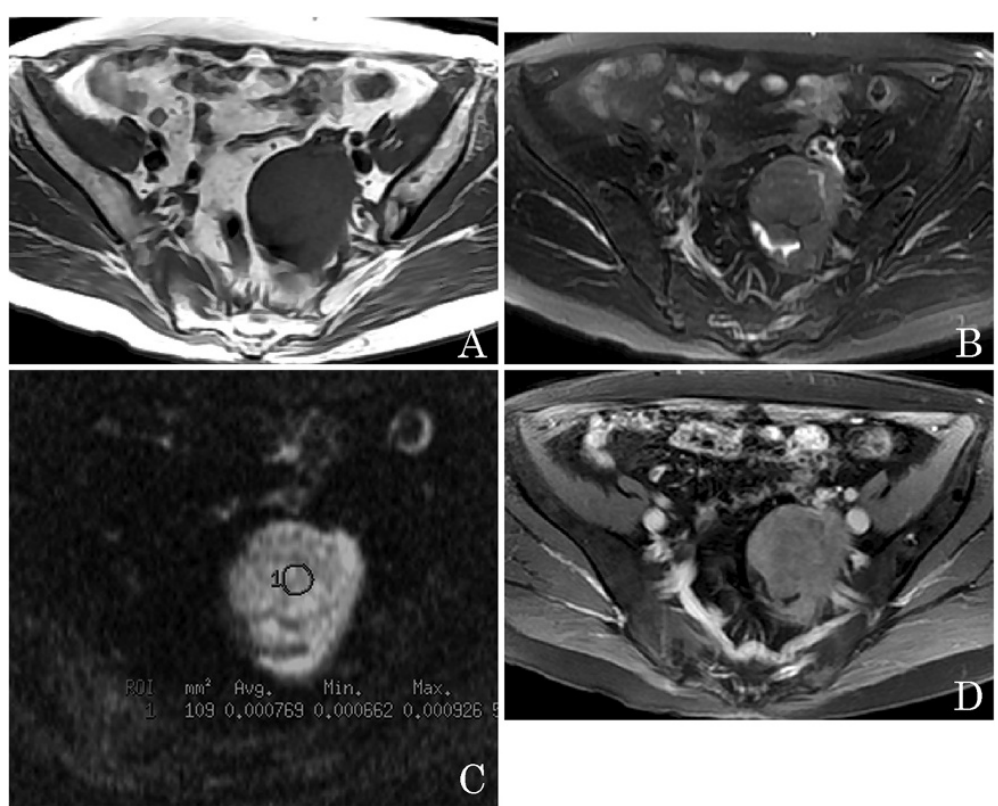

Figure 4 A 63-year-old woman with a left ovarian cystadenocarcinoma. (A) An axial T1-weighted image shows a left pelvic mass with isointensity. (B) Axial T2-weighted image reveals that the mass is a solid tumor with low signal intensity. (C) Axial diffusion-weighted imaging (DWI) obtained at $b=1,000 \mathrm{~s} / \mathrm{mm}^{2}$ reveals the solid tumor as hyperintense with a lower apparent diffusion coefficient (ADC) value (circle 1 , $A D C=0.769 \times 10^{-3} \mathrm{~mm}^{2} / \mathrm{s}$ ). (D) Axial contrast-enhanced T1-weighted image demonstrates marked enhancement of the solid part.

Furthermore, our study confirms that high signal intensity within solid components with low ADC values on DWI is a useful criterion for predicting malignancy. In our study, on T2-weighted images solid components of malignant tumors had high signal intensity in $84.4 \%$ and low signal intensity in $15.6 \%$. On the other hand, $100 \%$ of solid components in malignant lesions had high signal intensity on DW images with low ADC values. This finding, that of low signal intensity on T2-weighted images and high signal intensity on DWI in solid components, may result from a reduction in both the extracellular matrix and the diffusion space of water protons in the extracellular and intracellular dimensions due to an increased nuclear to cytoplasmic ratio and hypercellularity. However, the low signal intensity on T2-weighted images and DWI in benign ovarian tumors such as fibrothecomas, cystadenofibromas and Brenner tumors, may be due to the high density of fibers, the low cellularity,
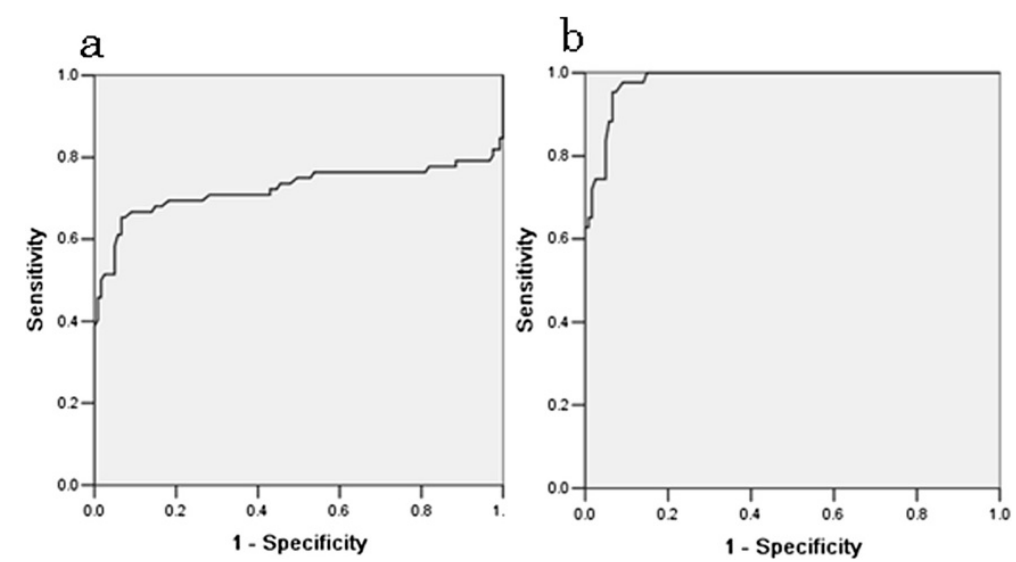

Figure 5 Comparative diagnostic values of quantitative apparent diffusion coefficient (ADC) value parameters for discriminating between benign and malignant ovarian tumors. When an absolute $A D C$ value less than $1.20 \times 10^{-3} \mathrm{~mm}^{2} / \mathrm{s}$ is predictive of malignancy, on receiver operating characteristic (ROC) analysis, the area under the curve (AUC) (a) is small (0.72), and when fibrothecomas, cystadenofibromas and Brenner tumors are excluded, the ROC AUC is the greatest (0.96), and thus it is probably the most effective factor for discriminating between benign and malignant ovarian masses (b). 
and the low water content in both extracellular and intracellular spaces [13-20]. Our study suggests that low signal intensity on T2-weighted images and DWI with low ADC values within a solid component is an effective criterion for predicting the presence of benign disease.

There were several limitations to our study. First, the exclusion of endometriomas and mature cystic teratomas and pure cystic adenomas may have induced a selection bias. As recommended by Takeuchi et al. [16], the DWI findings of endometriomas and mature cystic teratomas are problematic, and most cases can be accurately diagnosed on conventional MRI. Secondly, drawing the ROI on DW images while viewing T2-weighted or contrasted T-weighted images may have resulted in an information bias, as DWI usually has poor spatial resolution. A better approach would be to fuse DW images at $\mathrm{b}=1,000 \times 10^{-3} \mathrm{~mm}^{2} / \mathrm{s}$ onto structural images in order to accurately position the ROI of solid components that show an abnormal signal on DW images. Thirdly, the reported ADC threshold value needs to be validated in a larger group of patients, as many factors can affect the ADC value, including magnetic susceptibility, spatial resolution, signal to noise ratio, and the pathophysiological characteristics of the ovarian lesion.

\section{Conclusion}

DWI appears to be a useful method for differentiating between benign epithelial ovarian tumors with solid components and malignant ovarian tumors, and is associated with high sensitivity and specificity. The solid component within the complex adnexal mass that exhibits low signal intensity on T2-weighted images and DW images is invariably benign. In addition, a notable advantage of DWI is that it avoids further impairment of renal function, as the use of contrast medium is not necessary. For patients with renal failure, this would prevent the development of fibrosis.

\section{Competing interests}

This work had no specific funding. The authors declare that they have no conflict of interest.

\section{Authors' contributions}

WH Li planned, designed and analyzed the study, collected data and wrote the manuscript, YF Cui, G Ren collected data, P Zhang designed the study, XR Wu contributed as a pathologist and helped in writing the manuscript. All authors read and approved the final manuscript.

\section{Acknowledgements}

We thank Huarong Gong, Huitao AN, Qiufeng Yin, and Ming Liu for technical support.

\section{Author details}

'Department of Obstetrics and Gynecology, Xinhua Hospital affiliated to Shanghai JiaoTong University School of Medicine, Shanghai 200092, China. ${ }^{2}$ Department of Radiology, Xinhua Hospital affiliated to Shanghai JiaoTong University School of Medicine, Shanghai 200092, China. ${ }^{3}$ Department of
Pathology, Xinhua Hospital affiliated to Shanghai JiaoTong University School of Medicine, Shanghai 200092, China.

Received: 16 August 2012 Accepted: 18 October 2012

Published: 9 November 2012

\section{References}

1. Medeiros $L R$, Freitas $L B$, Rosa DD, Silva FR, Silva $L S$, Birtencourt $L T$, Edelweiss $\mathrm{Ml}$, Rosa Ml: Accuracy of magnetic resonance imaging in ovarian tumor: a systematic quantitative review. Am J Obstet Gynecol 2011, 204(67):e1-10.

2. Lalwani N, Prasad SR, Vikram R, Shanbhogue AK, Huettner PC, Fasih N: Histologic, molecular, and cytogenetic features of ovarian cancers: implications for diagnosis and treatment. Radiographics 2011, 31:625-646.

3. Bazot M, Nassar-Slaba J, Thomassin-Naggara I, Cortez A, Uzan S, Darai E: MR imaging compared with intraoperative frozen-section examination for diagnosis of adnexal tumors; correlation with final histology. Eur Radiol 2006, 16:2687-2699.

4. Timmerman D, Valentin L, Bourne TH, Collins WP, Verrelst $H$, Vergote $\mathrm{I}$ : Terms, definitions and measurements to describe the sonographic features of adenexal tumors: a consensus opinion from the International Ovarian Tumor Analysis (IOTA) Group. Ultrasound Obstet Gynecol 2000, 16:500-505.

5. Bazot M, Darai E, Nassar-Slaba J, Lafont C, Thomassin-Naggara I: Value of magnetic resonance imaging for the diagnosis of ovarian tumors: a review. J Comput Assist Tomogr 2008, 32:712-723.

6. Sohaib SAA, Sahdev A, Trappen PV, Jacobs IJ, Reznek RH: Characterization of adnexal mass lesions on MR imaging. AJR 2003, 180:1297-1304.

7. Ghossain MA, Buy JN, Ligneres C, Bazot M, Hassen K, Malbec L, Hugol D, Truc JB, Decroix Y, Poitout P: Epithelial tumors of the ovary: comparison of MR and CT findings. Radiology 1991, 181:863-870.

8. Jung SE, Lee JM, Rha SE, Byun JY, Jung Jl, Hahn ST: CT and MR imaging of ovarian tumors with emphasis on differential diagnosis. Radiographics 2002, 22:1305-1325.

9. Thomassin-Naggara I, Darai E, Cuenod CA, Rouzier R, Callard P, Bazot M: Dynamic contrast-enhanced magnetic resonance imaging: a useful tool for characterizing ovarian epithelial tumors. J Magn Reson Imaging 2008, 28:111-120.

10. Thomassin-Naggara I, Darai E, Cuenod CA, Fournier L, Toussaint I, Marsault C, Fournier L, Tossaint I, Marsault P, Bazot M: Contribution of diffusionweighted MR imaging for predicting benignity of complex adnexal masses. Eur Radiol 2009, 19:1544-1552.

11. Katayama M, Masui T, Kobayashi S, Ito T, Sakahara H, Nozaki A, Kabasawa H: Diffusion-weighted echo planar imaging of ovarian tumors: is it useful to measure apparent diffusion coefficients? J Comput Assist Tomogr 2002, 26:250-256

12. Li WH, Chu CT, Cui YF, Zhang P, Zhu MJ: Diffusion-weighted MRl: a useful technique to discriminate benign versus malignant ovarian surface epithelial tumors with solid and cystic components. Abdom Imaging 2012, 37:897-903.

13. Moteki T, Ishizaka H: Evaluation of cystic ovarian lesions using apparent diffusion coefficient from turboFLASH MR images. Br J Radiol 1998, 71:612-620.

14. Fujii S, Kakite S, Nishihara K, Kanasaki Y, Harada T, Kigawa J, Kaminou T, Ogawa T: Diagnostic accuracy of diffusion-weighted imaging in differentiating benign from malignant ovarian lesions. J Magn Reson Imaging 2008, 28:1149-1156.

15. Thomassin-Naggara I, Toussaint I, Perrot N, Rouzier P, Cuenod CA, Bazot M Darai ED: Characterization of complex adnexal masses: value of adding perfusion- and diffusion-weighted MR imaging to conventional MR imaging. Radiology 2011, 258:793-803.

16. Takeuchi M, Matsuzaki K, Nishitani: Diffusion-weighted magnetic resonance imaging of ovarian tumors: differentiation of benign and malignant solid components of ovarian masses. J Comput Assist Tomogr 2010, 34:173-176.

17. Li XC, Zhang WD, Zhu GB, Liu QG, Shen YC: Imaging features and pathologic characteristics of ovarian thecoma. J Comput Assist Tomogr 2012, 36:46-53.

18. Umemoto M, Shiota M, Shimono T, Hoshiai H: Preoperative diagnosis of ovarian tumors, focusing on the solid area based on diagnostic imaging. J Obstet Gynaecol Res 2006, 32:195-201. 
19. Erturk SM, Ichikawa T, Sano K, Motosugi U, Sou H, Araki T:

Diffusion-weighted magnetic resonance imaging for characterization of focal liver masses: impact of parallel imaging (SENSE) and $b$ value. J Comput Assist Tomogr 2008, 32:865-871.

20. Nakai G, Matsuki M, Inada Y, Tatsugami F, Tanikake M, Narabayashi I, Yamada T: Detection and evaluation of pelvic lymph nodes in patients with gynecologic malignancies using body diffusion-weighted magnetic resonance imaging. J Comput Assist Tomogr 2008, 32:764-768.

doi:10.1186/1477-7819-10-237

Cite this article as: Zhang et al:: Diagnostic accuracy of diffusionweighted imaging with conventional MR imaging for differentiating complex solid and cystic ovarian tumors at 1.5T. World Journal of Surgical Oncology 2012 10:237.

\section{Submit your next manuscript to BioMed Central and take full advantage of:}

- Convenient online submission

- Thorough peer review

- No space constraints or color figure charges

- Immediate publication on acceptance

- Inclusion in PubMed, CAS, Scopus and Google Scholar

- Research which is freely available for redistribution 